\title{
X-RAY IMAGES OF SUPERNOVA REMNANTS
}

\author{
Frederick D. Seward \\ Harvard-Smithsonian Center for Astrophysics \\ 60 Garden, Street, Cambridge, Massachusetts 02138
}

Einstein observations of supernova remnants have been reviewed and analyzed. Images of 44 galactic remnants have been reprocessed, merged when necessary, and collected into a catalog. Some bright remnants were viewed with both moderate and high resolution instruments (IPC with $1^{\prime}$ resolution and HRI with 4" resolution). Some IPC images of nearby remnants have been separated into 2 energy bands, $0.2-0.6 \mathrm{keV}$ and $0.6-4.5 \mathrm{keV}$; whereas most images cover the band $0.2-4.5 \mathrm{keV}$. The catalog consists of 72 images of the 44 remnants.

These images will be published in the form illustrated here. Contour levels are spaced geometrically as indicated below the figures and show the faintest observable features. The pictures are more linear and generally show only the brighter regions. Images are available now, however, in FITS format, on magnetic tape and may be obtained by writing to the author.

The $x$-ray morphology may be used to classify remnants. There are:

1) she1l-1ike SNR with definite $x$-ray limb brightening.

2) filled-center SNR, brightest at the center with little or no emission from the limb, $x$-ray spectra are suspected to be thermal, radio images are shell-like.

3) SNR with internal neutron star or bright synchrotron nebula indicating the presence of a hidden pulsar. Other data sometimes aid in this classification; e.g. a hard continuum $x$-ray spectrum or a center-bright radio morphology.

4) SNR dominated by a bright central source, probably an accretion-powered binary.

5) SNR with irregular morphology. None of the above categories apply, or data are too crude to determine the morphology.

The 44 remnants are listed here with a preliminary determination of the brightness as observed with the Einstein IPC.

Without the Einstein Observatory, these images of SNR would not exist. The observatory and imaging detectors were the result of the labors of R. Giacconi, H. Tananbaum, E. Schreier, L. Van Speybroeck, S. Murray, J.P. Henry, P. Gorenstein, F.R. Harnden, Jr., and D. Fabricant. Thanks also to J. Brody, M. O'Shaughnessy, and L Whitton who assisted in the preparation of material for this catalog. 
SNR Included in Catalog

\begin{tabular}{|c|c|c|c|c|c|}
\hline & Remnant & $\begin{array}{l}\text { Common } \\
\text { Name }\end{array}$ & $\underset{(\mathrm{c} / \mathrm{s})}{\text { IPC Rate }}$ & Type & Comments \\
\hline & $\frac{n e m i n d i n t}{4.5+6.8}$ & Kepler & $\frac{7.51}{7.3 \pm 0.4}$ & shell & SN 1604 \\
\hline & $6.4-0.1$ & W 28 & $3.2 \pm 0.4$ & & \\
\hline & $\begin{array}{l}11.2-0.3 \\
21.5-0.9\end{array}$ & & $\begin{array}{l}1.00 \pm 0.1 \\
0.49 \pm .05\end{array}$ & $\begin{array}{l}\text { Shell } \\
\text { synchrotron }\end{array}$ & \\
\hline & $27.4+0.0$ & Kes 73 & $1.07 \pm 0.1$ & central source & \\
\hline & $29.7-0.2$ & $\begin{array}{l}\text { Ke1838-049 } \\
\text { Kes } 75\end{array}$ & $0.22 \pm .03$ & synchrotron & not resolved \\
\hline 7 & $31.9+0.0$ & 3C 391 & $0.24 \pm .03$ & irregular & \\
\hline 8 & $33.7+0.0$ & Kes 79 & $0.44 \pm .05$ & irregular & \\
\hline & $34.6-0.5$ & W 44 & $3.3 \pm 0.3$ & full & \\
\hline & $\begin{array}{l}39.2-0.3 \\
39.7-2.0\end{array}$ & $\begin{array}{l}\text { C } 396 \\
\text { W } 50\end{array}$ & $\begin{array}{l}.06 \pm .01 \\
1.6 \text { ave. }\end{array}$ & $\begin{array}{l}\text { Ifreguiar } \\
\text { central source }\end{array}$ & \\
\hline $\begin{array}{l}11 \mathrm{~A} \\
12\end{array}$ & $41.1-0.3$ & $\begin{array}{l}\text { SS433 } \\
3 \text { C } 397\end{array}$ & $\begin{array}{l}1.2 \text { ave. } \\
0.75 \pm 0.1\end{array}$ & irregular & variable \\
\hline $\begin{array}{l}13 \\
14\end{array}$ & $43.3-0.2$ & W 49B & $0.67 \pm .06$ & full & \\
\hline $\begin{array}{l}14 \\
15\end{array}$ & $49.2-0.7$ & W 51 & 0.9 & irregular & \\
\hline & $65.3+5.7$ & GKP & $0.80 \pm 0.1$ & ruil & incomplete data \\
\hline $\begin{array}{l}17 \\
17\end{array}$ & $68.8+2.6$ & $\begin{array}{l}\text { CTB } 80 \\
1 \text { F1951+327 }\end{array}$ & $0.17 \pm .03$ & central source & \\
\hline $\begin{array}{l}18 \\
19\end{array}$ & $\begin{array}{l}74.3-8.5 \\
74.9+1.2\end{array}$ & $\begin{array}{l}\text { Cyg Loop } \\
\text { CTB } 87\end{array}$ & $\begin{array}{l}620 \pm 40 \\
.040 \pm .01\end{array}$ & $\begin{array}{l}\text { shell } \\
\text { synchrotron }\end{array}$ & \\
\hline 20 & $78.2+2.1$ & W 66 & $>0.6$ & & incomplete data \\
\hline & $82.2+5.3$ & W 63 & 0.3 & irregular & \\
\hline 22 & $109.2-1.0$ & $\begin{array}{l}\text { CTB } 109 \\
1 \mathrm{E} 2259+586\end{array}$ & $\begin{array}{l}5.2 \pm 0.4 \\
1.1 \pm 0.1\end{array}$ & central source & 7s period \\
\hline $\begin{array}{l}23 \\
24\end{array}$ & $\begin{array}{l}111.7-2.1 \\
119.5+9.8\end{array}$ & $\begin{array}{l}\text { Cas A } \\
\text { CTA } 1\end{array}$ & $\begin{array}{l}61 \pm 2 \\
0.9\end{array}$ & $\begin{array}{l}\text { shell } \\
\text { full }\end{array}$ & \\
\hline & $120.1+1.4$ & Tycho & $22.3 \pm 1$ & shell & SN 1572 \\
\hline 26 & $130.7+3.1$ & $3 \mathrm{C} 58$ & $0.35 \pm .04$ & synchrotron & \\
\hline & $132.7+1.3$ & HB 3, & 2.6 & irregular & \\
\hline $\begin{array}{l}28 \\
29\end{array}$ & $\begin{array}{l}160.4+2.8 \\
184.6-5.8\end{array}$ & $\begin{array}{l}\text { HB } 9 \\
\text { Crab }\end{array}$ & 684 & synchrotron & $\begin{array}{l}\text { incomplete data } \\
\text { SN } 1054\end{array}$ \\
\hline 29 & & PSR $0531+21$ & - & - & "age" $1240 \mathrm{yr}$ \\
\hline 30 & $189.0+3.0$ & IC 443 & 12.8 & irregular & \\
\hline & $260.4-3.4$ & Pup A & 230 & shell & \\
\hline 32 & $263.8-1.7$ & Vela SNR & 490 & irregular & \\
\hline 32 & $263.5-2.7$ & PSR 0833-45 & 2.1 & & "age" $13000 \mathrm{yr}$ \\
\hline 33 & 290.1-0.8 & MSH 11-61A & $0.47 \pm 0.1$ & full & \\
\hline 34 & $291.0-0.1$ & MSH 11-62 & $0.22 \pm .05$ & synchrotron & \\
\hline 35 & $292.0+1.8$ & MSH 11-54 & $9.1 \pm 1.0$ & irregular & \\
\hline & $296.5+10.0$ & PKS 1209-52 & & $\begin{array}{l}\text { Snell } \\
\text { shell }\end{array}$ & \\
\hline & $\begin{array}{l}315.4-2.3 \\
320.3-1.2\end{array}$ & $\begin{array}{l}\text { RCW 86 } \\
\text { MSH } 15-52\end{array}$ & $\begin{array}{l}8.5 \pm 1.0 \\
2.40 \pm 0.2\end{array}$ & $\begin{array}{l}\text { shell } \\
\text { synchrotron }\end{array}$ & \\
\hline 39 & & PSR 1509-58 & $0.30 \pm .04$ & & "age" 1550 yr \\
\hline 10 & $326.3-1.8$ & MSH 15-56 & 1.0 & irregular & \\
\hline & $327.1-1.1$ & & $.085 \pm .02$ & irregular & \\
\hline 2 & $327.4+0.4$ & Kes 27 & $0.40 \pm 0.1$ & full & \\
\hline & $\begin{array}{l}321.0+14.0 \\
332.4-0.4\end{array}$ & RCW 103 & $\begin{array}{l}11.1 \pm 1.0 \\
9.3 \pm 1.0\end{array}$ & shell & \\
\hline
\end{tabular}




\section{5+6.8 Kepler's SN}

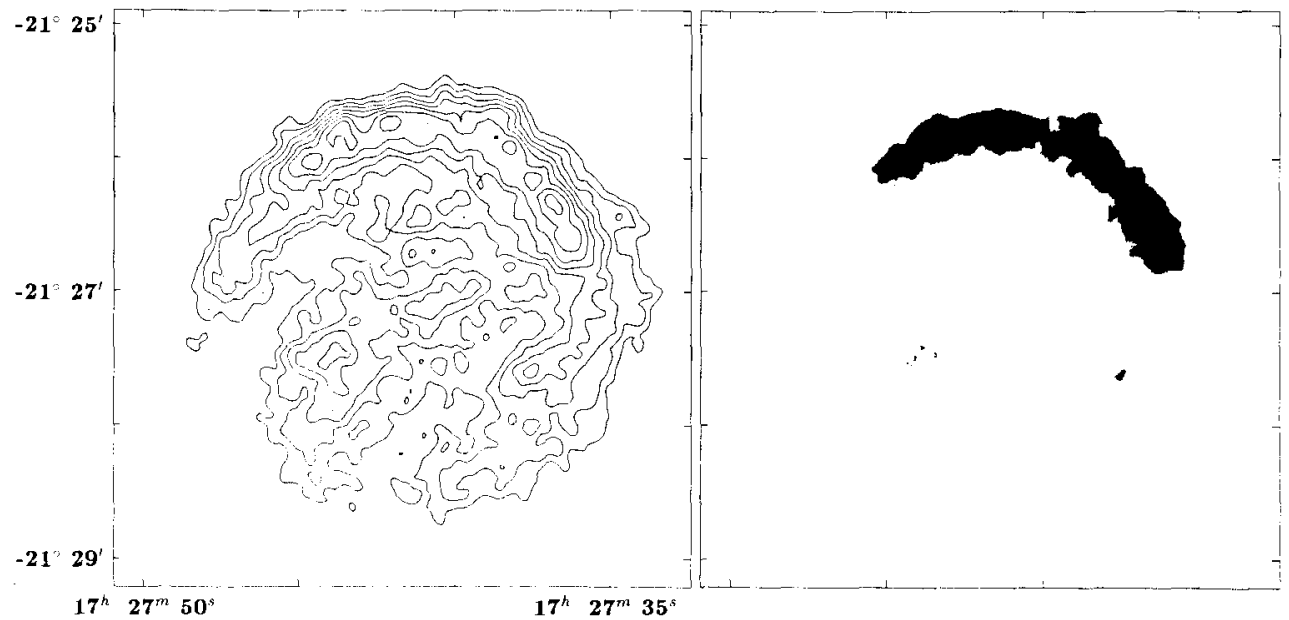

HRI, contour intervals are a factor of 1.3 in brightness.

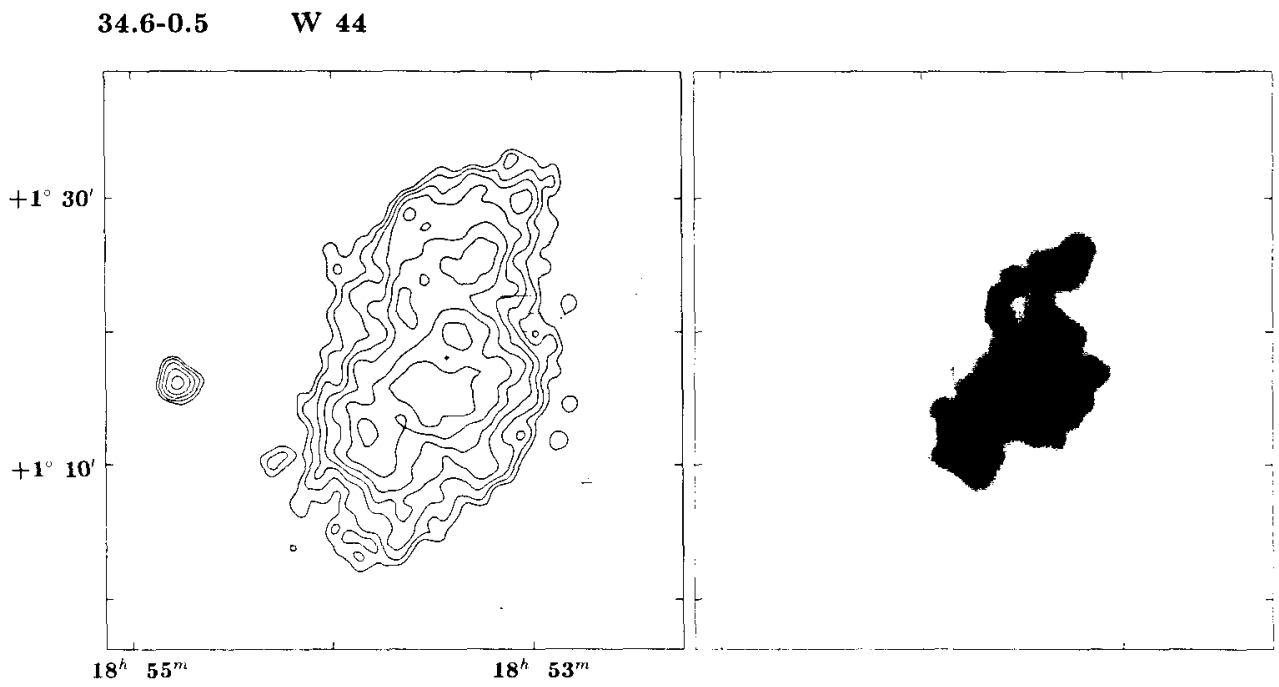

IPC, contour intervals are a factor of 1.4 in brightness. 


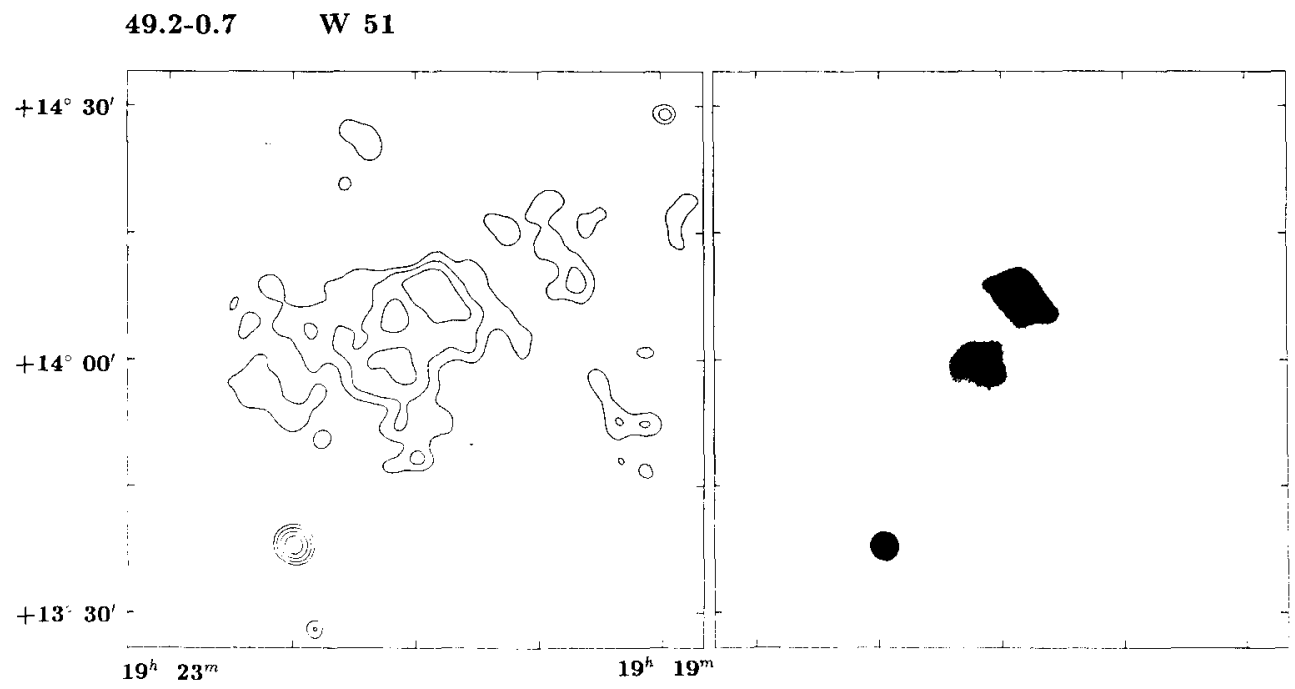

IPC, contour intervals are a factor of 1.5 in brightness.

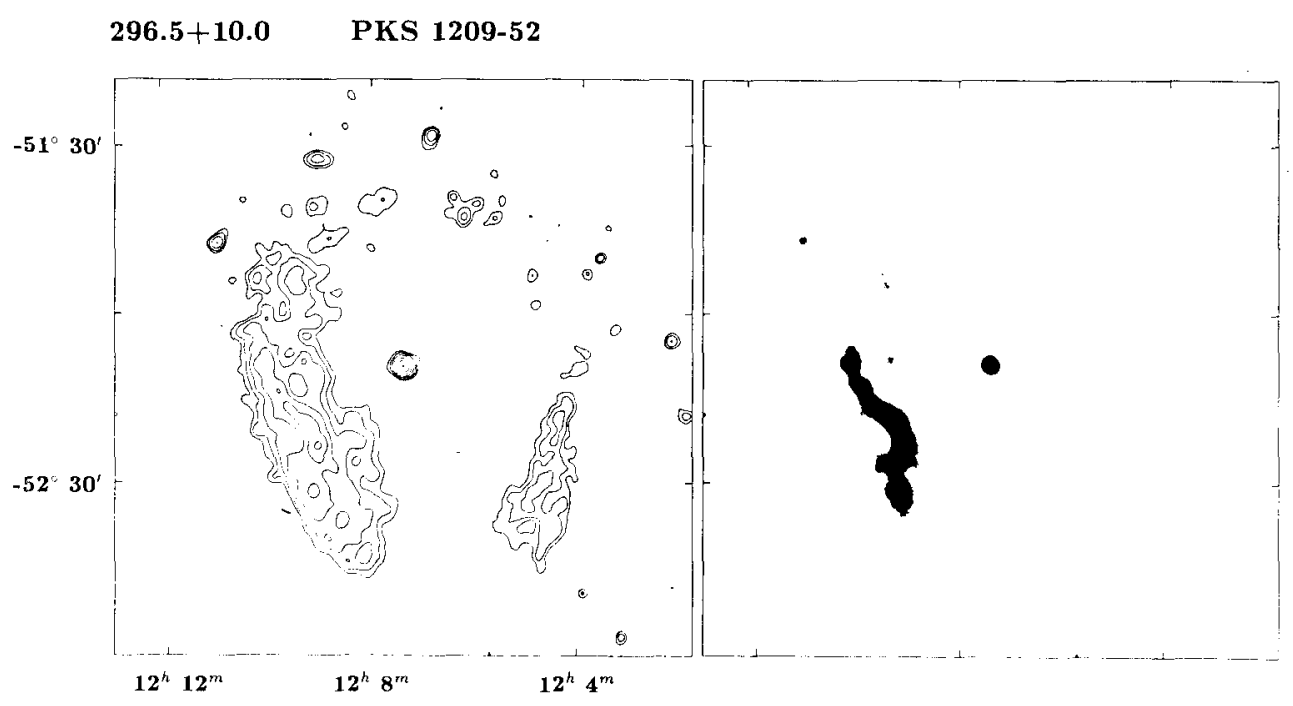

IPC, contour intervals are a factor of 1.5 in brightness. 